\title{
Exploitation des retenues et transport des sédiments
}

\author{
J. M. Cravero et P. Guichon \\ EDF - Service de la Production hydraulique
}

La connaissance de l'état de sédimentation des retenues d'EDF et l'amélioration des opérations d'évacuation des sédiments sont un souci permanent des exploitants du Service de la Production Hydraulique. Une synthèse des résultats de l'enquête de 1984 est présentée ci-après.

\section{Etat des retenues}

Sur les quatre-vingt-dix barrages fichés, vingt-neuf font l'objet d'un contrôle bathymétrique plus ou moins régulier et 21 font l'objet d'une estimation du volume des sédiments déposés. Les vidanges offrent la meilleure occasion d'évaluation ou de mesure, surtout par les possibilités de levées photogramétriques.

On rappelle qu'une crue exceptionnelle peut modifier profondément un régime d'alluvionnement établi depuis plusieurs décennies (Cas du CHASSEZAC après la crue de 1980).

Ces cinquante barrages représentent un volume de $3352 \mathrm{hm}^{3}$, le volume des sédiments déposés est estimé à $1044 \mathrm{~m}^{3}$ soit $6,1 \%$ de la capacité totale.

Sur les vingt barrages sédimentés à plus de $10 \%$, une réfection de la vidange de fond a été nécessaire. Pour cinq d'entre eux, la répartition de la sédimentation des retenues en fonction du pourcentage d'alluvionnement et de la capacité des réservoirs est représentée dans les deux graphiques ci-après :

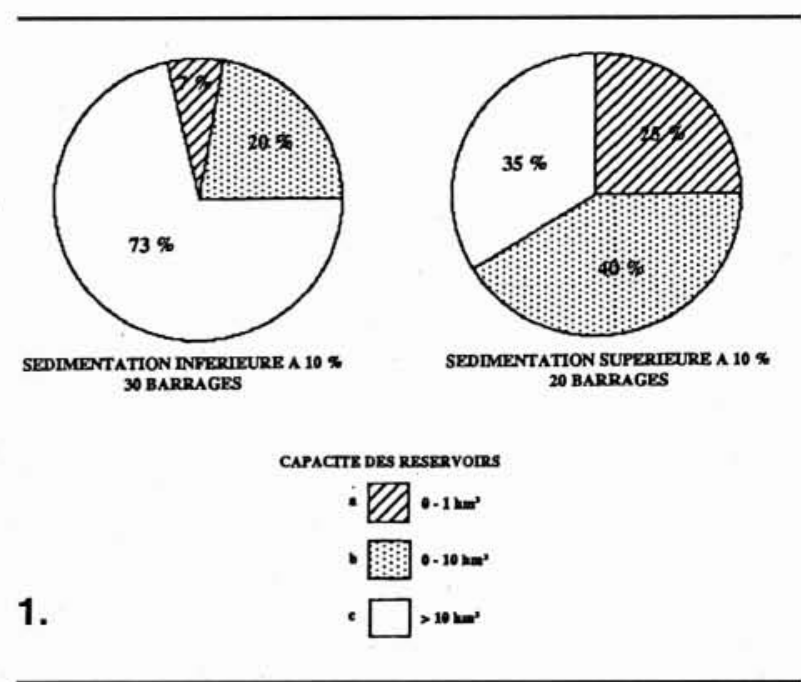

Trois familles de problèmes, déjà bien connus, apparaissent nettement :

- les importants dépôts minéraux de sable et de graviers dans les retenues des Alpes à moyenne altitude : SAUTET $16 \%$, Saint-Lazare $56 \%$ et L'Escale $75 \%$.

- la forte sédimentation organique des petites retenues du Massif Central, dont les rivières traversent des régions boisées (La Beaume, Saint-Nectaire, La Bourboule, Saillant, CoIndre).

- la sédimentation des parties d'eau morte du Rhin sur toutes les retenues immédiates en amont des barrages.

\section{Operation of reservoirs and sediment transportation}

The hydropower Generation Division of Electricite de France presents some results of its follow up of reservoir silting up, including the major problems that are encountered. On site experiences will be exposed such as evacuation of alluvions during the ten yearly drawdowns for inspection and outside those emptying periods. 
Au point de vue de la production d'énergie électrique, les réductions de capacité ne touchent, pour le moment, que les plus petits réservoirs, notamment ceux qui sont utilisés pour la régulation des débits.

\section{Vidanges décennales}

Sur les quatre-vingt-dix barrages, soixante ont fait l'objet d'au moins une vidange en général par visite décennale. Pour dix d'entre eux seulement, on a donné une estimation des volumes de sédiments évacués. Ce volume est, en général, faible sauf pour les retenues à la fois longues et encaissées.

Exemple de Thuries: $6,5 \mathrm{hm}^{3}$ sur le Viaur (Languedoc)

1983 est la première vidange complète depuis la mise en service en 1921.

Le programme de prévision d'abaissement du plan d'eau est très perturbé par des colmatages successifs des conduits de vidange conduisant à des élévations de la cote de la retenue et l'éboulement de talus de boues. Les conséquences ont été importantes pour les travaux programmés par EDF (augmentation de $50 \%$ des dépenses) et l'Environnement.

Malgré toutes les précautions prises, les conséquences physiques et chimiques à l'aval du barrage en période estivale et touristique a eu des répercussions notables et momentanées sur la qualité des eaux sur quelques dizaines de kilomètres.

Le suivi hydrobiologique de cette rivière a permis de conclure, 6 mois après, à un retour à la normale de la qualité physico-chimique de l'eau, du peuplement des macro-invertébrés benthiques et du peuplement piscicole.

Cette vidange ayant pourtant fait l'objet d'une préparation soignée, montre les difficultés rencontrées par l'exploitant pour maîtriser les éléments naturels pendant l'abaissement du plan d'eau.

\section{Opérations d'évacuation des sédiments hors période vidanges décennale}

Plusieurs régions ont des opérations d'évacuation, objet de consignes d'exploitation, approuvées ou en cours d'approbation par les différentes autorités concernées.

\subsection{En Savoie}

De nombreuses petites retenues font, jusqu'à présent, l'objet de chasses systématiques organisées dans le cadre d'une consigne d'exploitation approuvée par la Direction Régionale de l'Industrie et de la Recherche (Aigueblanche, Jotty, Mottets, Houches, Motz).

Compte tenu des caractéristiques de ces retenues $(0,3$ à $2 \mathrm{hm}^{3}$, un débit de crue important) et des possibilités des ouvrages d'évacuation, l'efficacité de ces chasses est remarquable ; malgré une sédimentation résiduelle importante, ces opérations permettent d'atteindre un relatif état d'équilibre.

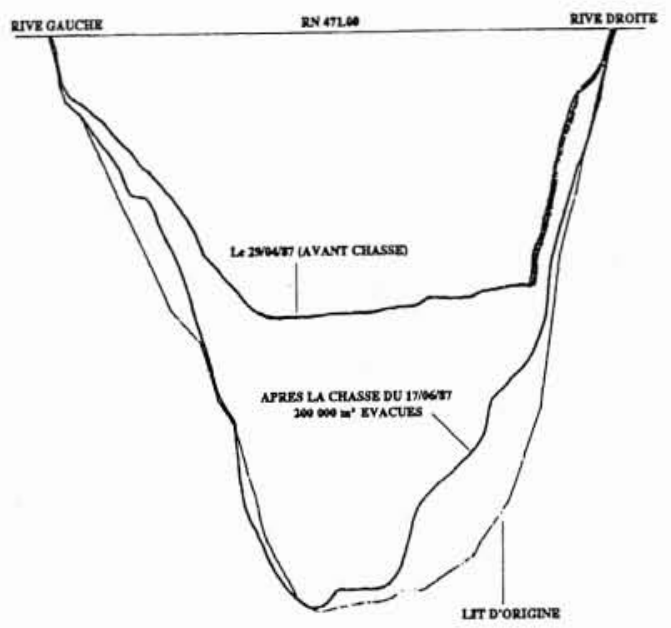

2. Exemple de la retenue d'Aigueblanche (Savoie). Mesures d'engravement en milieu de retenue.

\subsection{Sur la Basse Isère}

Les cinq barrages de faibles volumes (11,8-6-13,75-3,9 et $3,3 \mathrm{hm}^{3}$ ) des 70 derniers kilomètres font l'objet de vidanges triennales.

A partir d'essais et de mesures physico-chimiques, des consignes de manœuvres et de débits $\left(400 \mathrm{~m}^{3} / \mathrm{s}\right.$ sur l'Isère et $1200 \mathrm{~m}^{3} / \mathrm{s}$ sur le Rhône) ont été élaborées et sont en cours de validation pour les chasses.

L'efficacité de la démarche montre que l'Isère retrouve sa qualité d'origine après 48 heures. Il est à noter aucune mortalité piscicole depuis 1977.

Les matériaux solides transportés sont estimés entre 750000 et 1000000 tonnes pour une période d'effacement des barrages de 72 heures.

\subsection{Barrages de Castillon et d'Artigues (Pyrénées)}

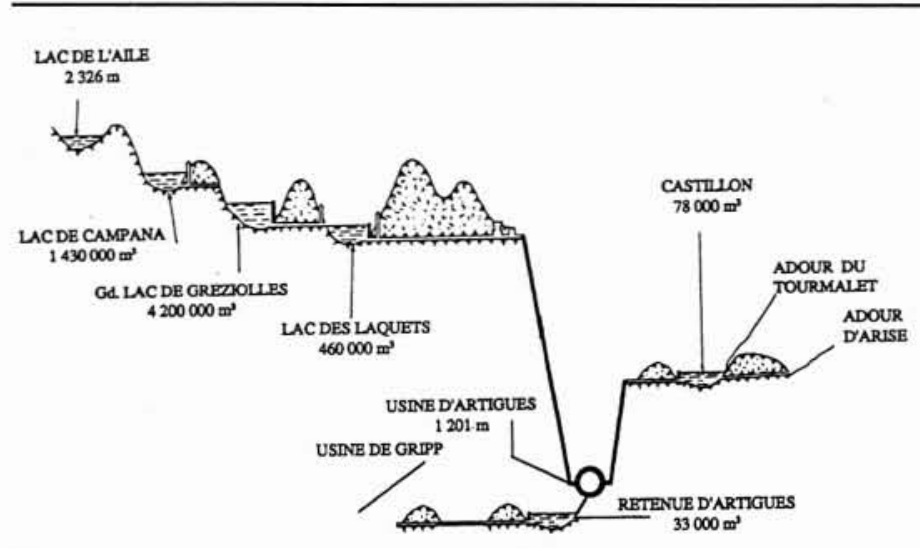

3. Castillon et Artigues. 
Les retenues de CASTILlon $\left(0,07 \mathrm{hm}^{3}\right)$ et ARTigues $\left(0,03 \mathrm{hm}^{3}\right)$ reçoivent chaque année des apports solides en quantité très importante se traduisant par un engravement général et un colmatage progressif des vannes de vidange et de prise d'eau.

Les vidanges de ces deux retenues sont de courte durée, 2 jours pour CASTILlon et 4 jours pour Artigues.

Le principe de la méthode est de maintenir à l'aval du barrage vidangé un taux des matières en suspension (MES) acceptable en constituant des réserves d'eau permettant d'augmenter la dilution.

\begin{tabular}{|l|c|c|}
\hline Bilan des sédiments évacués & 1987 & 1988 \\
\hline Castillon & $2000 \mathrm{~m}^{3}$ & $2000 \mathrm{~m}^{3}$ \\
\hline Artigues & $15000 \mathrm{~m}^{3}$ & $5000 \mathrm{~m}^{3}$ \\
\hline
\end{tabular}

Des opérations de désengravement mécanique ont également été essayées mais avec résultats préjudiciables pour la faune aquatique.

Les périodes optimales pour la réalisation des désengravements hydrauliques se situent à la période de forts débits (15 mai-15 juin).

L'expérience de ces dernières années partagée avec des partenaires constructifs devrait aboutir prochainement à l'établissement d'une procédure annuelle de vidange en période de crues.

\subsection{Barrage de Saint-Nectaire (Puy-de-Dôme)}

Petite retenue de $10000 \mathrm{~m}^{3}$ comblée à $90 \%$ en 1979 par des sédiments minéraux et organiques.

Pour éviter l'entraînement des alluvions pendant le dragage de la retenue (septembre 1979), un canal de dérivation de $140 \mathrm{~m}$ contournant le barrage a été creusé ( 8 jours de travail).

Le nettoyage de la retenue a duré 5 jours et aucune pollution n'a été constatée.

Le coût hors taxes en Francs 1988 est $236000 \mathrm{~F}$ pour $4000 \mathrm{~m}^{3}$ de vase déplacée.

Après les crues de janvier et décembre 1982, la majorité du dépôt d'alluvions s'est reconstitué.

La solution mise en œuvre en 1979, économiquement très lourde pour ce type d'aménagement, peut être très rapidement remise en cause par une crue importante.

En résumé, le curage des petites retenues doit être précédé d'une étude sur les mécanismes de l'alluvionnement pouvant conduire à l'adaptation du mode d'exploitation en période de crue.

\subsection{Le Rhin}

Le Rhin, lors des hautes eaux, est relativement chargé en matières en suspension. Ce sont les zones amont et aval des écluses et l'amont des barrages qui sont le plus affectées par l'envasement.
La politique suivie depuis 1970 jusqu'à nos jours a été de draguer les différents aménagements. Les matériaux sont généralement des sédiments très fins et très collants nécessitant l'utilisation de bennes preneuses, grapins ou de suceuses munies d'un désagrégateur.

On notera que les volumes, enlevés en 15 à 20 ans, sont du même ordre de grandeur que les dépôts stockés et sur les seuls 200 premiers mètres à l'amont des barrages ; cela donne une image des limites des moyens mécaniques.

Depuis 1970, le volume dragué représente en moyenne $93000 \mathrm{~m}^{3}$ par an se répartissant environ par moitié à l'amont des barrages et dans les garages amont et aval des écluses.

Les sédiments sont mis en dépôt à terre par voie hydraulique. L'eau utilisée s'infiltre progressivement et les dépôts se consolident généralement dans un délai d'un an.

La saturation des zones de dépôts possibles se produira inéluctablement et des essais de rejets dans le courant sont en cours et laissent penser que cette méthode pourrait être retenue.

\section{La Durance}

L'aménagement de la Durance s'est achevé en 1978. C'est au total 18 centrales hydrauliques dont 15 sur la Durance et 3 sur le Verdon.

Les barrages de SERRE-PonçON (d'une capacité de $\left.1270 \mathrm{hm}^{3}\right)$ sur la Durance et de SAINTE-Croix $\left(770 \mathrm{hm}^{3}\right)$ sont les deux principales retenues.

La vocation de l'ensemble est hydroélectrique et agricole.

Les études réalisées préalablement à la construction ont permis d'évaluer à $126000 \mathrm{~m}^{3} / \mathrm{an}$ les transports par charriage des gros matériaux au niveau de SERRE-PONÇON et $140000 \mathrm{~m}^{3} / \mathrm{an}$ à l'aval de CADARACHE.

Les mesures effectuées de 1951 à 1960 pour les matériaux en suspension donnent des volumes moyens annuels de 1,5 million de $\mathrm{m}^{3}$ à SERRE-PONÇON et 2,5 millions de $\mathrm{m}^{3}$ à CADARACHE.

Les mesures effectuées ont permis de constater que la moitié des tonnages d'apports solides étaient concentrés sur quelques jours dans l'année. Il a été convenu d'arrêter les usines de SALON et SAINT-CHAMAS dans ces périodes-là et de restituer les eaux dans le cours naturel de la Durance.

Ces mesures ont été complétées par la construction d'un bassin de 122 ha de décantation à CADARACHE retenant $65 \%$ des apports limoneux se déversant normalement dans l'Etang de Berre.

\section{La situation actuelle}

SERRE-PonçON: Le phénomène de sédimentation de la retenue est légèrement supérieur aux prévisions mais n'est pas un phénomène préoccupant (diminution de $2 \%$ de la retenue énergétique utile en 25 ans). Seule la queue de retenue laisse apparaître des dépôts de limon pour un niveau inférieur de 5 mètres au niveau normal. 


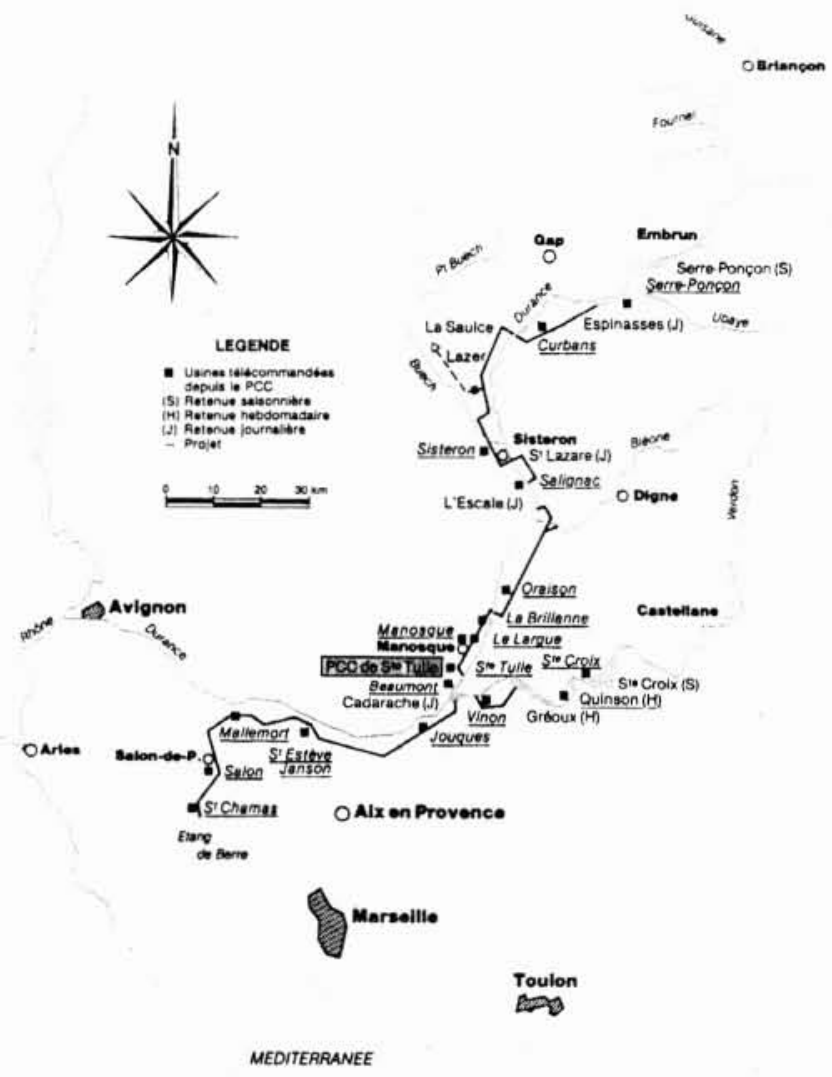

4. Centrales de la Durance et du Verdon commandées depuis le PCC.

En descendant le cours de la Durance, nous rencontrons successivement les retenues suivantes:

ESPINASSE : elle ne soulève aucune préoccupation.

LA SAULCE: La sédimentation est notable mais sans conséquence pour l'environnement.

\section{SAINT-LAZARE et L'Escale :}

Saint-Lazare: Mise en service en 1975, elle est comblée au $3 / 4$ dix ans après avec une certaine stabilisation des volumes déposés $\left(4\right.$ millions de $\mathrm{m}^{3}$ ). Des études en cours par le Laboratoire national d'hydraulique et la Division technique générale d'EDF laissent supposer des solutions pouvant conduire à modifier l'exploitation du lac de SERRE-PonCon, aménager les zones envasées par des plantations et cultures, remodeler ponctuellement le terrain pour créer des écoulements préférentiels. La solution dragage ne peut être retenue, elle conduirait à étaler sur 500 hectares une épaisseur de 4 mètres de vase.

L'Escale: Mise en service en 1963, est comblée après 23 ans à $75 \%$, soit 13 millions de $\mathrm{m}^{3}$ de sédiments.

CADARAChe: Sa fonction de décanteur est bien assurée (la turbidité de sortie n'a pratiquement jamais dépassé $1 \mathrm{~g} / \mathrm{l})$, compte tenu des quantités retenues, le comblement du bassin interviendrait dans une vingtaine d'années.

L'ETANG DE BERRE : un sujet de préoccupation.

Après 20 ans d'exploitation, le principe du détournement de la Durance dans l'Etang de Berre reste très contesté en raison des transformations écologiques qui sont: le volume annuel d'eau douce et les tonnages de sédiments.

Aucune étude n'a permis à ce jour de séparer de façon nette l'impact de ces deux facteurs.

La construction d'un nouveau bassin à la sortie de l'usine de Beaumont réduira, de façon importante, les quantités de limons envoyées dans l'Etang de Berre.

D'autres solutions sont envisageables mais nécessitent de fixer les objectifs de développement de l'Etang de Berre (tourisme, pêche, industries, etc...) et d'étudier les poids respectifs des différents paramètres (eau douce, sédiments. pollutions organiques et industrielles) dans la modification de l'équilibre écologique de l'Etang.

\section{Conclusions}

La variété des situations rencontrées lors des vidanges montre que les solutions utilisées sont rarement généralisables.

Mais l'accumulation des diverses expériences, le développement de la bathymétrie périodique, de la mesure des matières en suspension totale (MEST) et autres mesures physiques dans les débits évacués en cours de vidange permettent progressivement d'affiner la politique du Service de la Production hydraulique et d'améliorer les méthodes d'exploitation de retenues vis-à-vis de la sédimentation et des opérations de vidanges ou de chasses. 